\title{
Dynamics of an SIS Model on Homogeneous Networks with Delayed Reduction of Contact Numbers
}

\author{
Maoxing Liu* ${ }^{*}$ and Gergely Röst ${ }^{\dagger}$ \\ * Department of Mathematics \\ North University of China, Taiyuan, Shanxi, P. R. China, 030051 \\ Email: liumaoxing@126.com \\ $\dagger$ Bolyai Institute \\ University of Szeged, Szeged, Hungary \\ Email: rost@math.u-szeged.hu
}

Received: 12 July 2012, accepted: 4 October 2012, published: 27 December 2012

\begin{abstract}
During infectious disease outbreaks, people may modify their contact patterns after realizing the risk of infection. In this paper, we assume that individuals make the decision of reducing a fraction of their links when the density of infected individuals exceeds some threshold, but the decision is made with some delay. Under such assumption, we study the dynamics of a delayed SIS epidemic model on homogenous networks. By theoretical analysis and simulations, we conclude that the density of infected individuals periodically oscillate for some range of the basic reproduction number. Our results indicate that information delays can have important effects on the dynamics of infectious diseases.
\end{abstract}

Keywords-epidemic model; networks; delay; stability; oscillation

\section{INTRODUCTION}

A great deal of research in mathematical epidemiology has been done for complex networks during the past years [1], [2], [3], [4], [5], [6], [7], [8], [9], [10], [11], [12]. These works focus mostly on two different complex networks: the Watts-Strogatz model and the Barabási-Albert model. The first model is a relatively homogeneous network exhibiting small-world properties [1], and the second one is a typical example of a scalefree network [2]. On these networks, the SIS model is generally used to study the spread of infections via connections between the nodes of the networks.

In disease transmission models, time delay plays an important role in many epidemiological mechanisms. Various delayed epidemic models have been extensively studied (see, for example, Refs. [13], [14], [15], [16], [17], [18], [19]). Recently there have been some results about delay epidemic models on complex networks. In the paper [20], the authors present a modified SIS model with the effect of time delay in the transmission on small-world and scale-free networks. They found that the presence of the delay may enhance outbreaks and increase the prevalence of infectious diseases in these networks. Whereas in [21], the authors consider a delayed SIR epidemic model on uncorrelated complex network and addressed the effect of time lag on the shape and number of epidemic waves. They showed that even when the transmission rate is below the critical threshold, a larger delay can cause that the disease takes off while the force of infection is not increased. Otherwise, a large delay can cause multiple waves with larger amplitudes in the second and subsequent waves.

In fact, on one hand, during infectious disease outbreaks, individuals may reduce their activities after receiving information about the risk of infection. For 
example, people will reduce the time that they go out, students will not attend school, and so on, and such information on the ongoing epidemics may impact the dynamics itself [22], as the contact network changes [23]. Social distancing can be used as a control measure as well. On the other hand, due to the latent period of diseases, the concealment of infected individuals, or the time needed for collecting and analyzing epidemic data, the exact number of infected individuals is hard to be known in real time. Based on the above facts, in this paper we assume that individuals adjust their connections according to some information delay concerning the actual disease prevalence. For simplicity, we work with the assumption that individuals uniformly and randomly reduce the number of their connections by some factor whenever the density of infected individuals exceeds a given threshold. We study the effects of time-delayed information on the decision-making of individuals, and then we study the dynamics of the spread of the disease under such circumstances. By theoretical analysis and simulations, we conclude that the disease will be eradicated under some conditions, or tend to an endemic state, but it can also oscillate in a periodic pattern when the structure of networks and the time delay are properly given. All these behaviors are completely characterized by the basic reproduction number.

The paper is organized as follows. In the following section, we set up a delayed SIS model on homogenous networks and analyse the existence of equilibria. We also give some results on stability, permanence and oscillation of the disease in Section 2. In Section 3, some numerical simulations illustrating the key points of the theoretical analysis are given. At the last section we offer a discussion of our results.

\section{Model And AnAlysis}

\section{A. The Model}

In this section, we consider a susceptible-infectedsusceptible (SIS) model on homogenous networks. In the SIS model, infectious (I) individuals contaminate their susceptible (S) neighbors with some transmission rate. Meanwhile, infected individuals recover at some rate and return to the susceptible state again. By using the mean-field approach on homogenous networks, in [6] the authors arrived to the following epidemic model:

$$
\frac{d I(t)}{d t}=-\mu I(t)+\beta\langle k\rangle I(t)(1-I(t)) .
$$

Here $I(t) \in[0,1]$ denotes the density of infected nodes at time $t$. The first term considers infected nodes recovering with rate $\mu$. The second term of the right-hand side of Eq. (1) represents the newly infected nodes. This is proportional to the transmission rate $\beta$, the number of links emanating from each node $\langle k\rangle$, and the probability that a given link points to a healthy node, which is $1-I(t)$. Here $\mu, \beta,\langle k\rangle$ are positive constants.

We suppose that individuals will reduce their links according to the information they learn on the disease spread. If the disease is not widespread, people remain in contact with others as usual. With the increasing number of the infected individuals, people reduce their activities and temporarily terminate some of their links. We assume this is governed by the following function:

$$
h(I)= \begin{cases}1, & I \leq p, \\ q, & I>p\end{cases}
$$

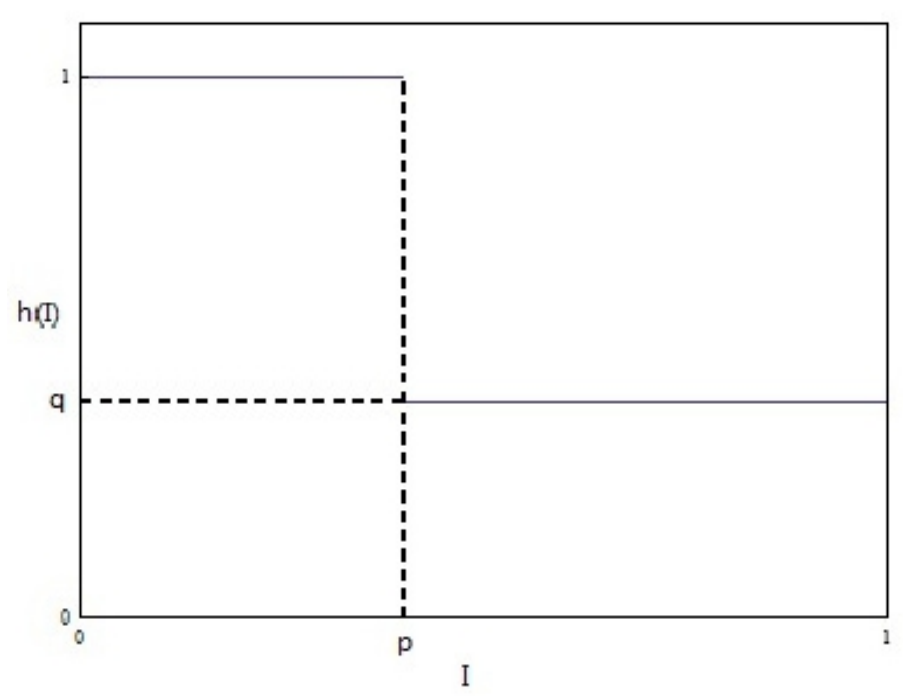

Fig. 1. The graph of $h(I)$.

where $0<p, q<1$. As shown in Fig. 1. when $I \leq p$ the number of links of individuals are the same as usual $\langle k\rangle$; when $I>p$ (i.e. the density of infectious nodes exceeds the threshold $p$ ), the links of individuals are reduced to a lower level $q\langle k\rangle$. By assuming a time delay $\tau>0$ in making this reduction, we obtain the following epidemic model with discontinuous right hand side:

$$
\frac{d I(t)}{d t}=-\mu I(t)+\beta\langle k\rangle h(I(t-\tau)) I(t)(1-I(t)) .
$$

For the sake of simplicity, we rescale time by $\tilde{I}(t)=$ $I\left(\mu^{-1} t\right)$, then writing the equation for $\tilde{I}(t)$ and dropping the tilde to use the notation $I(t)$ for the variable in the 
rescaled time, Eq. (2) is transformed into

$$
\frac{d I(t)}{d t}=-I(t)+R_{0} h(I(t-\tau)) I(t)(1-I(t)),
$$

where $R_{0}=\frac{\beta\langle k\rangle}{\mu}$ is the basic reproduction number, which expresses the number of secondary infections generated by a single infected node in a fully susceptible homogenous network. Eq. (3) is a scalar delay differential equation, which are known to be able to exhibit complicated behaviour if the nonlinearity is nonmonotone [24]. The technical complications due to the discontinuity of the right hand side can be avoided if by a solution of Eq. (3) we mean a continuous function $I(t)$ satisfying

$I(t)=I(t-\tau)+\int_{t-\tau}^{t} I(s)\left(R_{0} h(I(s-\tau))(1-I(s))-1\right) d s$

for all $t \geq \tau$. Throughout the paper by $I^{\prime}(t)$ we mean the right derivative when $I(t-\tau)=p$, this will not cause any confusion. Given the interpretation of the model, we only consider solutions $I(t) \in[0,1]$. Clearly solutions from this interval remain in $[0,1]$ for all future time. For the existence of the equilibria, we have the following statement.

\section{Proposition 2.1.}

(a) The disease free equilibrium $I_{0}^{*}=0$ always exists,

(b) if $1<R_{0} \leq \frac{1}{1-p}$, Eq. $\sqrt{3}$ has a positive equilibrium $I_{1}^{*}$,

(c) if $R_{0}>\frac{1}{q(1-p)}$, Eq. 3 has a different positive equilibrium $I_{2}^{*}$,

(d) if $R_{0}<1$ or $\frac{1}{1-p}<R_{0}<\frac{1}{q(1-p)}$, there is no positive equilibrium.

Proof. To obtain the equilibria of Eq. (3), we set $I(t) \equiv I(t-\tau) \equiv I^{*}$, and let the right hand side of Eq. (3) be zero, so

$$
R_{0} h\left(I^{*}\right) I^{*}\left(1-I^{*}\right)=I^{*} .
$$

First we have $I^{*}=0$, which corresponds to the disease free equilibrium. If $I^{*} \neq 0$, then from Eq. (4) we get

$$
R_{0} h\left(I^{*}\right)\left(1-I^{*}\right)=1 .
$$

This does not have a positive solution for $R_{0}<1$. If $R_{0}>1$, we distinguish two cases. When $I^{*} \leq p$, we have $I^{*}=1-\frac{1}{R_{0}}$ (let's denote it by $I_{1}^{*}$ ), and when $I^{*}>p$, we have $I^{*}=1-\frac{1}{q R_{0}}$ (denoted by $I_{2}^{*}$ ). To satisfy $I_{1}^{*} \leq p$, we obtain $1-\frac{1}{R_{0}} \leq p$, which is $R_{0} \leq \frac{1}{1-p}$. Similarly, $I_{2}^{*}>p$ is equivalent to $R_{0}>\frac{1}{q(1-p)}$.

Proposition 2.2. If $R_{0}<1$, then $I_{0}$ is globally asymptotically stable. If $R_{0}>1$, then $I_{0}$ is unstable.
Proof. If $R_{0}<1$, the statement easily follows from the comparison

$$
\frac{d I(t)}{d t} \leq I(t)\left[R_{0}-1\right]
$$

As

$$
\frac{d I(t)}{d t}=I(t)\left[R_{0}-1\right]
$$

is the linear variational equation around zero, the disease free equilibrium is unstable if $R_{0}>1$.

\section{B. Permanence}

For the permanence of Eq. (3), we find the following result.

Theorem 2.3: If $R_{0}>1$, system (3) is permanent. More precisely,

$1-\frac{1}{q R_{0}} \leq \liminf _{t \rightarrow \infty} I(t) \leq \limsup _{t \rightarrow \infty} I(t) \leq 1-\frac{1}{R_{0}}$.

Proof: First we show $I_{\infty}=\liminf _{t \rightarrow \infty} I(t) \geq 1-\frac{1}{q R_{0}}$. Suppose that $0<I\left(t_{1}\right) \leq 1-\frac{1}{q R_{0}}$ for some $t_{1}$. Thus, by the definition of $h$,

$$
\left(1-I\left(t_{1}\right)\right) R_{0} h\left(I\left(t_{1}-\tau\right)\right) \geq 1
$$

and from Eq. (3) we have

$I^{\prime}\left(t_{1}\right)=I\left(t_{1}\right)\left(-1+R_{0} h\left(I\left(t_{1}-\tau\right)\right)\left(1-I\left(t_{1}\right)\right)\right) \geq 0$, that is to say, the solution at $t_{1}$ is increasing, and since by Proposition 2.1. a positive equilibrium less than $1-\frac{1}{q R_{0}}$ can not exist, we obtain

$$
I_{\infty} \geq 1-\frac{1}{q R_{0}} .
$$

Next we show $I^{\infty}=\limsup _{t \rightarrow \infty} I(t) \leq 1-\frac{1}{R_{0}}$. Using similar argument, supposing $I\left(t_{2}\right) \geq 1-\frac{1}{R_{0}}$ for some $t_{2}$, we obtain

$$
\left(1-I\left(t_{2}\right)\right) R_{0} h\left(I\left(t_{2}-\tau\right) \leq 1,\right.
$$

and from Eq. (3) we have

$I^{\prime}\left(t_{2}\right)=I\left(t_{2}\right)\left(-1+R_{0} h\left(I\left(t_{2}-\tau\right)\right)\left(1-I\left(t_{2}\right)\right)\right) \leq 0$.

The solution at $t_{2}$ is decreasing, and by the nonexistence of equilibrium greater than $1-\frac{1}{R_{0}}$, we obtain

$$
I^{\infty} \leq 1-\frac{1}{R_{0}} .
$$

The graph showing the relation of $I^{*}$ and $R_{0}$ is depicted in Fig.2. From Fig.2, we can also see the effect of the structure of the network. By increasing the average degree $\langle k\rangle, R_{0}$ is also increasing, and the equilibrium will change from zero to nonzero, then to oscillation, then back to a nonzero equilibrium again; thus system (3) will experience different dynamical behaviors. 


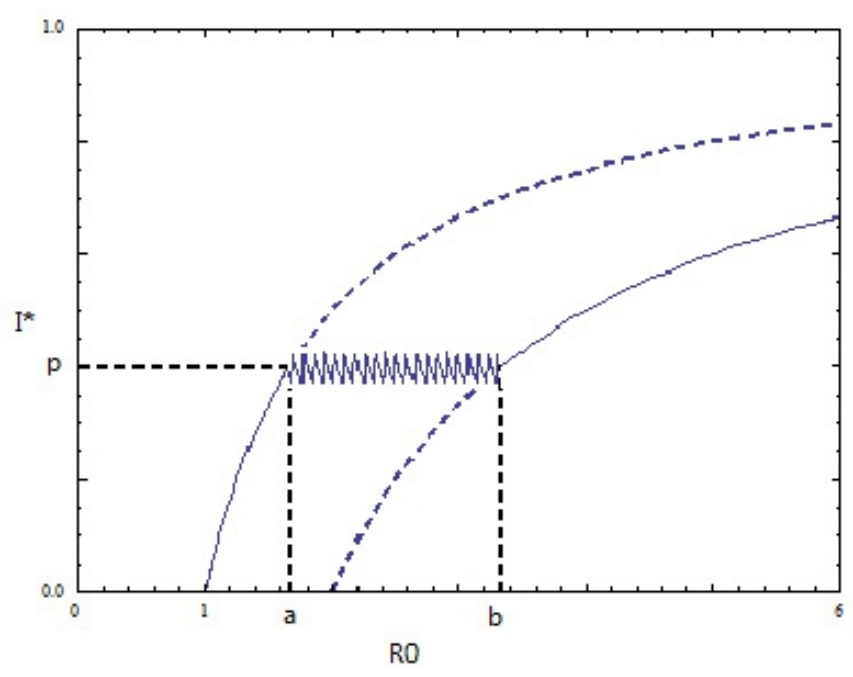

Fig. 2. The relation of $I^{*}$ and $R_{0}$, here $a=\frac{1}{1-p}, b=\frac{1}{q(1-p)}$. The solutions are oscillating when $a<R_{0}<b$.

\section{Stability and Oscillation}

For the stability of the positive equilibria and oscillations of solutions, we have the following results.

Proposition 2.4: For Eq. (3), the following claims are true:

(a) If $1<R_{0}<\frac{1}{1-p}$, the positive equilibrium $I_{1}^{*}$ is locally asymptotically stable.

(b) If $R_{0}>\frac{1}{q(1-p)}$, the positive equilibrium $I_{2}^{*}$ is locally asymptotically stable.

Proof: (a) If $1<R_{0}<\frac{1}{1-p}$, Eq. $\sqrt{3}$ has a positive equilibrium $I_{1}^{*}=1-\frac{1}{R_{0}}<p$, thus we can choose a small neighborhood of $I_{1}^{*}$ such that $I(t)<p$ as long as the solution is in this neighborhood. Thus, restricted to this neighborhood $h \equiv 1$ and Eq. (3) becomes an ordinary differential equation, and we can easily obtain that the positive equilibrium $I_{1}^{*}$ is locally asymptotically stable. Using the same argument, we can prove (b).

Theorem 2.5: If $\frac{1}{1-p}<R_{0}<\frac{1}{q(1-p)}$, then all solutions of Eq. (3) oscillate around $p$.

Proof: Let $\frac{1}{1-p}<R_{0}<\frac{1}{q(1-p)}$, and suppose that a solution $I(t)$ of Eq. (3) does not oscillate around $p$, then there must exist $T_{1}$ such that

$$
I(t) \leq p, \quad \text { for } \quad t \geq T_{1}
$$

or a $T_{2}$ such that

$$
I(t) \geq p, \quad \text { for } \quad t \geq T_{2} .
$$

In the following we show that none of them is possible. If $I(t) \leq p$ for $t \geq T_{1}$, we have

$$
I^{\prime}(t)=-I(t)+R_{0} I(t)(1-I(t))
$$

for $t \geq T_{1}+\tau$. In this case the solution $I(t)$ converges to $1-\frac{1}{R_{0}}>p$, which is a contradiction.

Similarly, if $I(t)>p$ for $t$ larger than some $T_{3}$, we have

$$
I^{\prime}(t)=-I(t)+q R_{0} I(t)(1-I(t))
$$

for $t \geq T_{2}+\tau$. In this case the solution $I(t)$ converges to $1-\frac{1}{q R_{0}}<p$, this is also a contradiction. We finish by demonstrating that if $I(t) \geq p$ for all $t>T_{2}$, then there is a $T_{3}$ such that $I(t)>p$ for all $t>T_{3}$. If that is not true, then we can find arbitrarily large $t_{k}$ such that $I\left(t_{k}\right)=p$. If $I\left(t_{k}-\tau\right)>p$, then $I^{\prime}$ is continuous at $t_{k}$ and $I^{\prime}\left(t_{k}\right)=0$ must hold, but then $h\left(I\left(t_{k}-\tau\right)\right)=q$ and so

$$
I^{\prime}\left(t_{k}\right)=p\left(R_{0} q(1-p)-1\right) \neq 0 .
$$

To exclude the remaining case when $I\left(t_{k}-\tau\right)=p$, we impose the additional assumption that on some interval of length $\tau$ our solution takes the value $p$ at most finitely many times, then this property of $I(t)$ will be inherited to any interval $[t-\tau, t]$ for future $t$ by the integral representation. By integration we also obtain

$\frac{I\left(t_{k}\right)}{I\left(t_{k}-\tau\right)}=\exp \left[\int_{t_{k}-\tau}^{t_{k}} R_{0} h(I(s-\tau))(1-I(s))-1 d s\right]$,

which implies that the integral in the exponent should be zero. However, $R_{0} h(I(s-\tau))(1-I(s))-1 \leq 0$ whenever $I(s-\tau)>p$ and $h(I(s-\tau))=q$. By our assumption the equality $I(s-\tau)=p$ holds on a set of measure zero, thus the integral is negative, which is a contradiction.

\section{Simulations}

In this section, we discuss some examples and simulations. Our purpose is to illustrate the sharpness of the results of the previous section. Here we set initial data as constant functions. First we set $p=0.8, q=0.6$ and demonstrate the stability of the zero equilibrium, as shown in Fig. 3 with $R_{0}=0.8$.

In the case $R_{0}=2$, as shown in Fig.4, the positive equilibrium $I_{1}^{*}$ is asymptotically stable. While for $R_{0}=3.6, p=0.5, q=0.4$ and $\tau=1.2$, as shown in Fig.5, solutions of Eq.(3) are oscillatory. In the case $R_{0}=2.7, p=0.1, q=0.6$, as shown in Fig.6, the positive equilibrium $I_{2}^{*}$ is asymptotically stable.

If $\frac{1}{1-p}<R_{0}<\frac{1}{q(1-p)}$, then all solutions of Eq. (3) oscillate around $p$. In this case, to illustrate the effect of the time delay, we consider distinct values of the delay while other parameters are fixed. In the first case the delay is small, depicted in Fig. 7, with $\tau=0.3$, and the amplitude of the solution around $p$ is apparently small. 


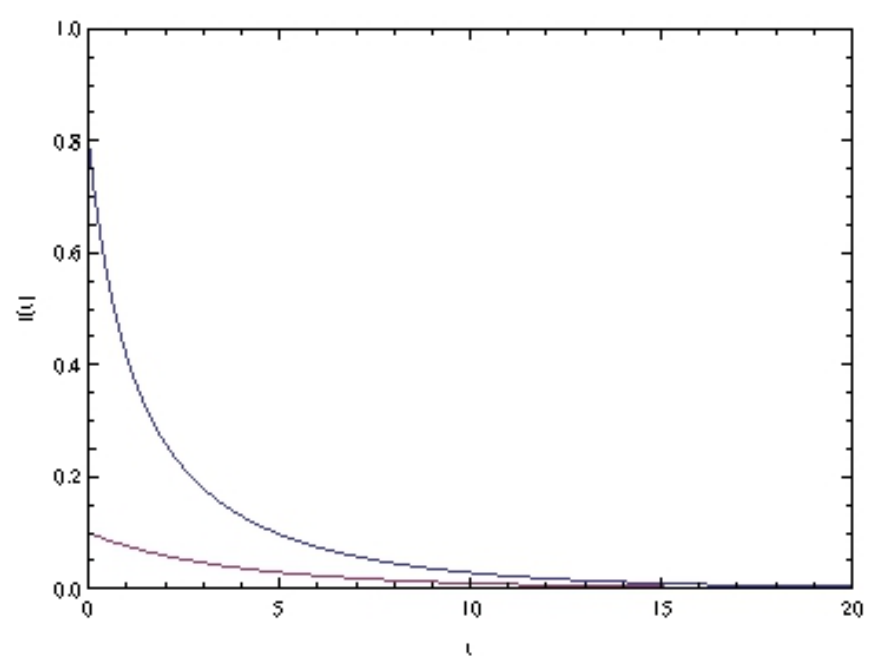

Fig. 3. The time evolutions of the density of infection with $I(0)=$ 0.1 and $0.8, R_{0}=0.8, p=0.8, q=0.6$, and $\tau=0$.

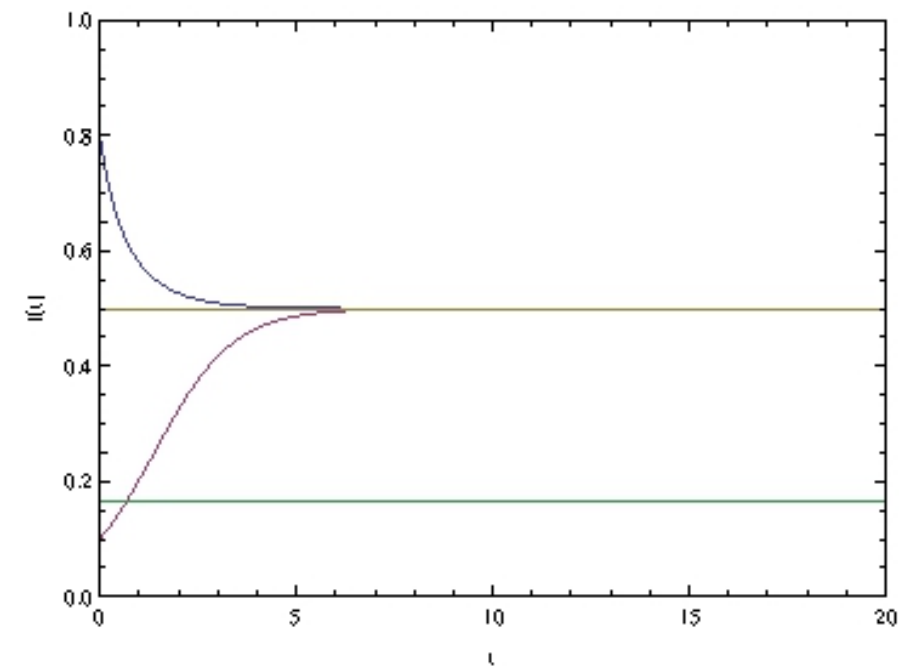

Fig. 4. The time evolutions of the density of infection with $I(0)=$ 0.1 and $0.8, R_{0}=2, p=0.8, q=0.6$ and $\tau=0$.

In the second case, if the delay is larger than in the first case $(\tau=3.6)$, we observe increased amplitude in Fig. 8. However, for all delays, the solutions always oscillate between $1-\frac{1}{R_{0}}$ and $1-\frac{1}{q R_{0}}$. These values are represented by the straight lines in Fig. 7 and Fig. 8 and one can see that the bounds are rather sharp for large delays.

\section{CONCLUSION}

In this paper, we studied a delayed model for an SIS epidemic process in a population of individuals on a homogenous network. We assumed that individuals temporarily reduce the number of their links by a factor

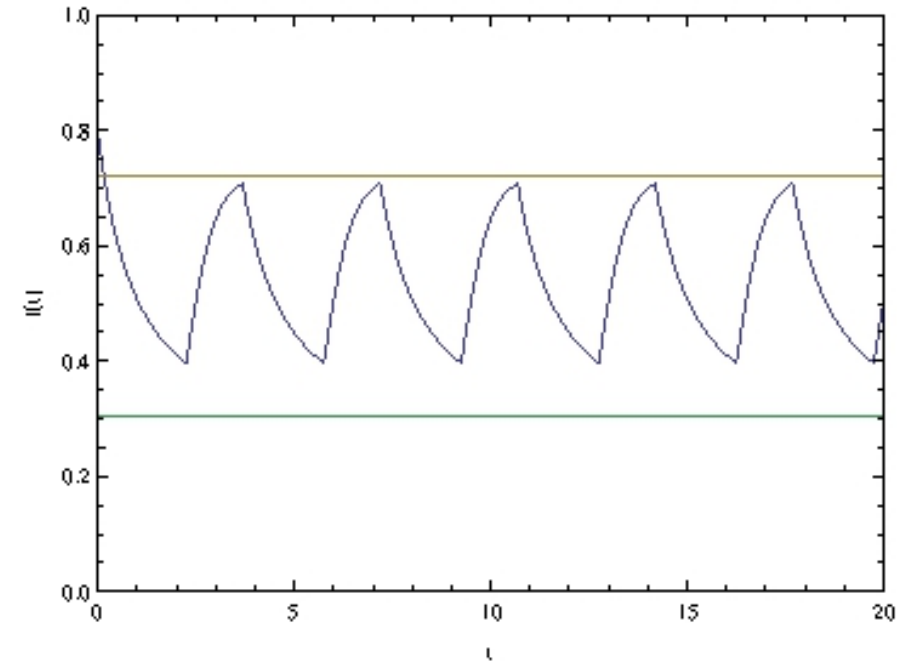

Fig. 5. The time evolutions of the density of infection with $I(0)=$ $0.8, R_{0}=3.6, p=0.5, q=0.4$ and $\tau=1.2$.

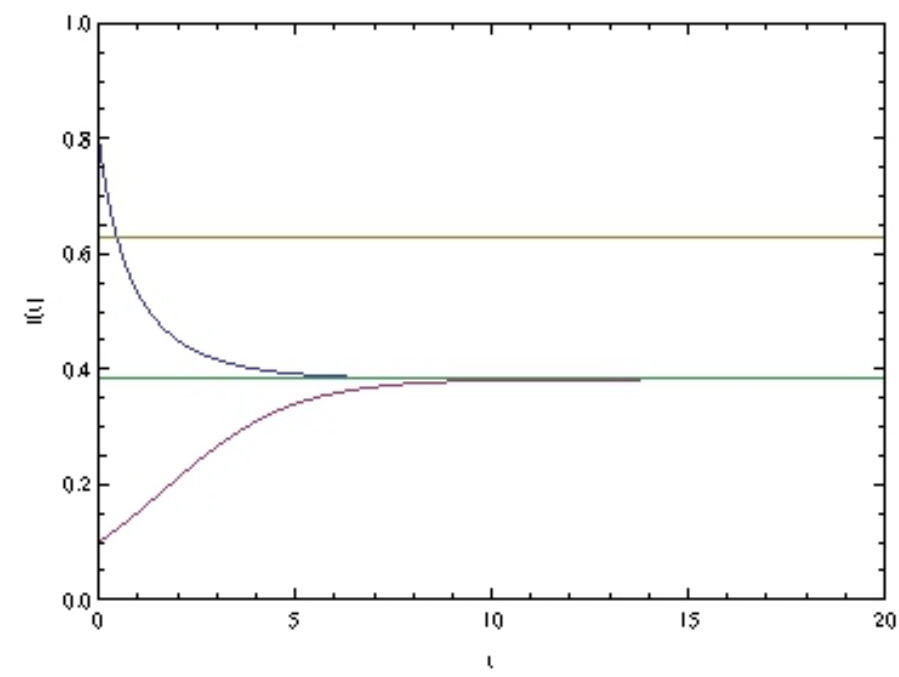

Fig. 6. The time evolutions of the density of infection with $I(0)=$ 0.1 and $0.8, R_{0}=2.7, p=0.1, q=0.6$ and $\tau=0$.

$q$ when the density of infections exceeds the threshold number $p$, but this modification in the contact pattern is done with some delay $\tau$. When the basic reproduction number is smaller than one, the disease will be eradicated. For reproduction numbers larger than one, we showed that the disease persists in the population. If the endemic state is lower than the threshold, then the reduction of contacts will never be triggered and the solution converges to an endemic equilibrium $I_{1}^{*}$. If there is an endemic state even with the reduction of contacts which is at higher level than the threshold, then the terminated links remain inactive for all future time and 


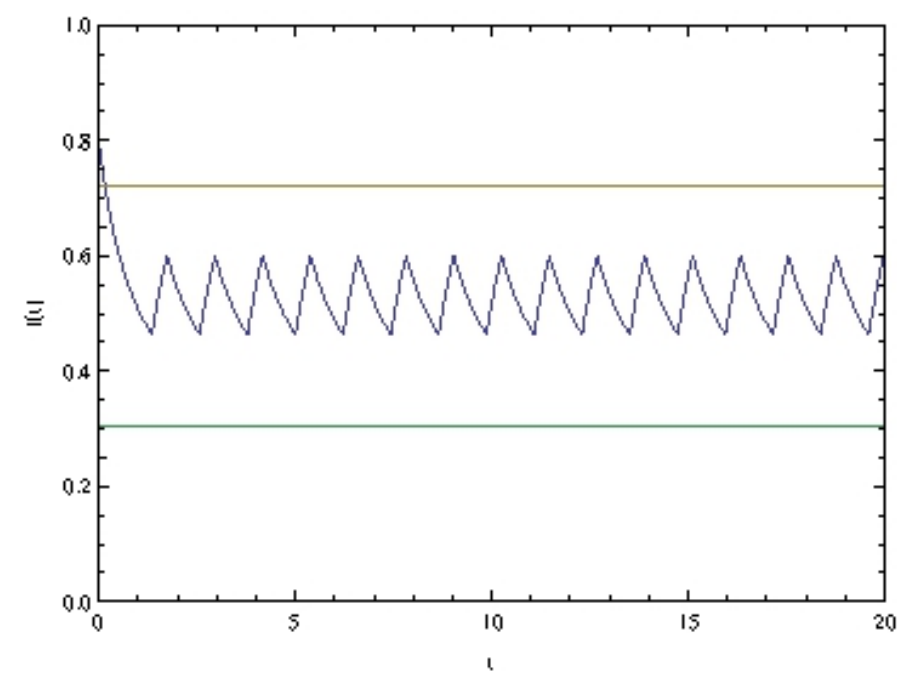

Fig. 7. The time evolutions of the density of infection with $I(0)=$ $0.8, R_{0}=3.6, p=0.5, q=0.4$ and $\tau=0.3$.

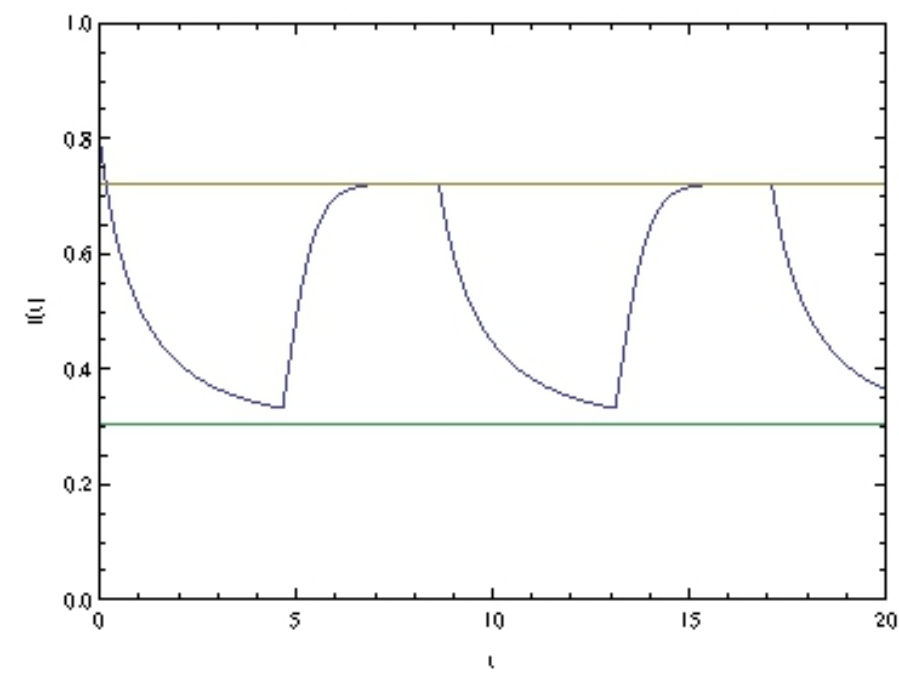

Fig. 8. The time evolutions of the density of infection with $I(0)=$ $0.8, R_{0}=3.6, p=0.5, q=0.4$ and $\tau=3.6$.

the solution converges to a different endemic state $I_{2}^{*}$. There is an interesting intermediate situation though for a range of basic reproduction numbers, when the reduction is triggered, but with such reduced transmission the endemic state is below the threshold, thus the links will be reactivated again, which helps the disease to spread more, thus triggering the reduction and so on, forming an interesting periodic oscillatory pattern. The time delay has significance in determining the characteristics of this oscillation: longer delay leads to larger amplitudes. Our results indicate that the structure of the network (which influences the reproduction number), the threshold type reduction in contacts and the delayed decision in reduction interestingly interplay on influencing the spreading dynamics of infectious diseases.

\section{ACKNOWLEDGMENT}

The authors would like to thank the support of National Sciences Foundation of China (10901145), Top Young Academic Leaders of Higher Learning Institutions of Shanxi, European Research Council Starting Investigator Grant Nr. 259559, Hungarian Scientific Research Fund OTKA K75517, and Bolyai Scholarship of Hungarian Academy of Sciences.

\section{REFERENCES}

[1] D. J. Watts and S. H. Strogatz, "Collective dynamics of 'smallworld' networks," Nature, vol. 393, pp. 440-442, 1998. http://dx.doi.org/10.1038/30918

[2] A. L. Barabasi and R. Albert, "Emergence of Scaling in Random Networks", Science, vol. 286, pp. 509-512, 1999. http://dx.doi.org/10.1126/science.286.5439.509

[3] R. M. May and A. L. Lloyd, "Infection dynamics on scale-free networks", Phys. Rev. E, vol. 64, 066112, 2001.

[4] Y. Moreno, R. Pastor-Satorras, A. Vespignani, "Epidemic outbreaks in complex heterogeneous networks", Eur. Phys. J. B, vol. 26, pp. 521-529, 2002. http://dx.doi.org/10.1140/epjb/e20020122

[5] M. E. J. Newman, "Spread of epidemic disease on networks", Phys. Rev. E, vol. 66, 016128, 2002.

[6] R. Pastor-Satorras and A. Vespignani, "Epidemic dynamics and endemic states in complex networks", Phys. Rev. E, vol. 63, 066117, 2001.

[7] X. C. Fu, M. Small, D. M. Walker, H. F. Zhang, "Epidemic dynamics on scale-free networks with piecewise linear infectivity and immunization", Phys. Rev. E, vol. 77, 036113, 2008.

[8] M. X. Liu and J. Ruan, "Modelling the spread of sexually transmitted diseases on scale-free networks", Chin. Phys. B, vol. 18 , pp. 2115-2120, 2009. http://dx.doi.org/10.1088/1674-1056/18/6/002

[9] A. d'Onofrio, "A note on the global behaviour of the networkbased SIS epidemic model", Nonlinear Anal.: RWA, vol. 9, pp. 1567-1572, 2008. http://dx.doi.org/10.1016/j.nonrwa.2007.04.001

[10] L. Wang and G. Z. Dai, "Global stability of virus spreading in complex heterogeneous networks", SIAM J. Appl. Math., vol. 68, pp.1495-1502, 2008. http://dx.doi.org/10.1137/070694582

[11] J. Lou and T. Ruggeri, "The dynamics of spreading and immune strategies of sexually transmitted diseases on scale-free network", J. Math. Anal. Appl., vol. 365, pp. 210-219, 2010. http://dx.doi.org/10.1016/j.jmaa.2009.10.044

[12] I. Z. Kiss, P. L. Simon, R. R. Kao, "A contact-network-based formulation of a preferential mixing model", Bull. Math. Biol., vol. 71, pp. 888-905, 2009. http://dx.doi.org/10.1007/s11538-008-9386-2

[13] F. Zhang, Z. Z. Li, F. Zhang, "Global stability of an SIR epidemic model with constant infectious period", Appl. Math. Comput., vol. 199, pp. 285-291, 2008. http://dx.doi.org/10.1016/j.amc.2007.09.053 
[14] J. Z. Zhang, Z. Jin, Q. X. Liu, Z. Y. Zhang, "Analysis of a delayed SIR model with nonlinear incidence rate", Discrete Dyn. Nat. Soc., pp. 1-16, 2008. http://dx.doi.org/10.1155/2008/636153

[15] R. Xu and Z. Ma, Stability of a delayed SIRS epidemic model with a nonlinear incidence rate, Chaos, Soliton. Fract., vol. 41, pp. 2319-2325, 2009.

[16] A. Kaddar, "On the dynamics of a delayed SIR epidemic model with a modified saturated incidence rate", Elec. J. of Diff. Equ., vol. 133, pp. 1-7, 2009.

[17] G. Röst and J. Wu, "SEIR epidemiological model with varying infectivity and infinite delay", Math. Biosci. Eng., vol. 5, pp. 389-402, 2008.

http://dx.doi.org/10.3934/mbe.2008.5.389

[18] M. E. Alexander, S. M. Moghadas, G. Röst, J. Wu, "A Delay Differential Model for Pandemic Influenza with Antiviral Treatment”, Bull. Math. Biol, vol. 70, pp. 382-397, 2008. http://dx.doi.org/10.1007/s11538-007-9257-2

[19] G. Röst, Sh. Y. Huang, L. Székely, "On a SEIR epidemic model with delay”, Dynam. Systems and Appl., vol. 21, pp. 33-48, 2012.
[20] X. Xu, H. Peng, X. Wang, Y. Wang, "Epidemic spreading with time delay in complex networks", Physica A, vol. 367, pp. 525530, 2006 http://dx.doi.org/10.1016/j.physa.2005.11.035

[21] S. Zou, J. Wu, Y. Chen, "Multiple epidemic waves in delayed susceptible-infected-recovered models on complex networks", Phys. Rev. E, vol. 83, 056121, 2011.

[22] I. Z. Kiss, J. Cassell, M. Recker, P. L. Simon, "The impact of information transmission on epidemic outbreaks", Math. Biosci., vol. 225, pp. 1-10, 2010. http://dx.doi.org/10.1016/j.mbs.2009.11.009

[23] I. Z. Kiss, L. Berthouze, T. J. Taylor, P. L. Simon, "Modelling approaches for simple dynamic networks and applications to disease transmission models", Proc. Roy. Soc. A, vol. 468, pp. 1332-1355, 2012 http://dx.doi.org/10.1098/rspa.2011.0349

[24] G. Röst, J. Wu, "Domain-decomposition method for the global dynamics of delay differential equations with unimodal feedback", Proc. R. Soc. A, vol. 463, pp. 2655-2669, 2007. 